\title{
SUMO wrestling in cell movement
}

\author{
Miia M Rytinki ${ }^{1}$, Jorma J Palvimo ${ }^{1}$ \\ ${ }^{1}$ Institute of Biomedicine, University of Eastern Finland, P.O. Box 1627, FI-70211 Kuopio, Finland \\ Cell Research (2011) 21:3-5. doi:10.1038/cr.2010.162; published online 23 November 2010
}

Small ubiquitin-like modifier (SUMO) proteins 1,2 and 3 can be covalently conjugated to specific lysine residues on target proteins in a process dubbed SUMOylation. This conserved posttranslational modification that was reported for the first time in 1996 has emerged as an important regulatory mechanism in cell physiology, especially in nuclear signaling, transport, transcription and DNA replication/ repair $[1,2]$. SUMOylation is thought to provide docking sites for interacting proteins, which leads to altered cellular localization, activity and/or stability of the target proteins. The SUMOylation process is similar to ubiquitylation, but requires a distinct enzymatic $\mathrm{E} 1 \rightarrow \mathrm{E} 2 \rightarrow \mathrm{E} 3$ cascade. The SUMOs activated by the SAE1/2 heterodimer (E1) are transferred to the UBC9 (E2) that conjugates them to target lysines. The conjugation process is enhanced by SUMO E3 ligases. Protein Inhibitor of Activated STAT (PIAS) 1 and 3 proteins were originally identified as repressors of STAT signaling. However, the four PIAS family members 1,2, 3 and 4 play a wide role in cell regulation also by promoting target-specific SUMOylation and hence by functioning as SUMO E3 ligases [3]. SUMOylation is a reversible modification reversed by a family of SUMO-specific proteases, SENPs.

Although, the SUMOylation machin-

Correspondence: Jorma J Palvimo

E-mail: jorma.palvimo@uef.fi ery, its targets as well as PIAS-interacting proteins are enriched in the nucleus, there are rare examples of cytoplasmic SUMOylated proteins that interact with PIAS proteins, such as septins and PIAS ortholog Siz1 in yeast bud neck and PIAS3 and metabotropic glutamate receptor-8 in mammalian cells $[4,5]$. Recent isolation of Rho-like GTPase Rac1 as a PIAS3-interacting protein by Castillo-Lluva and co-workers adds a prominent and intriguing new entity to the list of cytoplasmic proteins functionally regulated by SUMOylation [6]. The group used a cell culture and proteomics-based approach to identify proteins that interact with Rac1 in response to cell migration that was stimulated by hepatocyte growth factor (HGF). Rac1 is a member of the evolutionary conserved Rho/Rac subfamily of the Ras superfamily of GTPases. It is an intracellular transducer that plays a key role in the regulation of cytoskeleton dynamics and organization and cell migration [7, 8]. Rac1 can also influence cell cycle and transcriptional dynamics. Cell migration is a central mechanism in the development and maintenance of healthy multicellular organisms [9]. The migration that is often initiated by an extracellular signal is a multistep process that consists of alterations in the cytoskeleton, cell adhesions and extracellular matrix. Mesenchymal-type of migration is characterized by an elongated cell shape. It requires extracellular proteolysis and engagement of integrin and it additionally relies on Rac1-medi- ated cell polarization and lamellipodia formation [9]. Therefore, it is hardly surprising that deregulated Rho/Rac signaling can contribute to a wide range of diseases, including cancer.

Rac1 was previously known to be regulated through several mechanisms, including control of nucleotide exchange (guanine nucleotide exchange factors, GEFs, activating Rac1) and hydrolysis (GTPase-activating proteins, GAPs, inactivating Rac1), and regulation of subcellular localization by posttranslational prenylation [10]. Interestingly, PIAS3 was found to interact primarily with the activated Rac1-GTP complex [6]. Although the PIAS3 is mainly found in the nucleus, small amounts of PIAS3 are also localized in the cytoplasm. Activation of cell migration after HGF exposure resulted in relocalization of PIAS3 from the cytoplasm to the cell membrane, where it colocalized with the Rac1-GTP (Figure 1). Rac1 was conjugated to SUMO-1 in response to the HGF treatment and the SUMO-1 modification was enhanced by PIAS3. The importance of PIAS3 in the Rac1 activation and function was shown by RNA interference. Depletion of cellular PIAS3 markedly reduced Rac1 activation, cell migration and impaired lamellipodia-membrane ruffle formation. The induction of Rac1 SUMOylation was not restricted to HGF signaling, but is a more general mechanism in the activation of Rac1, as it was also seen upon calcium-switched cell adhesion. One enigma in protein SUMOylation 

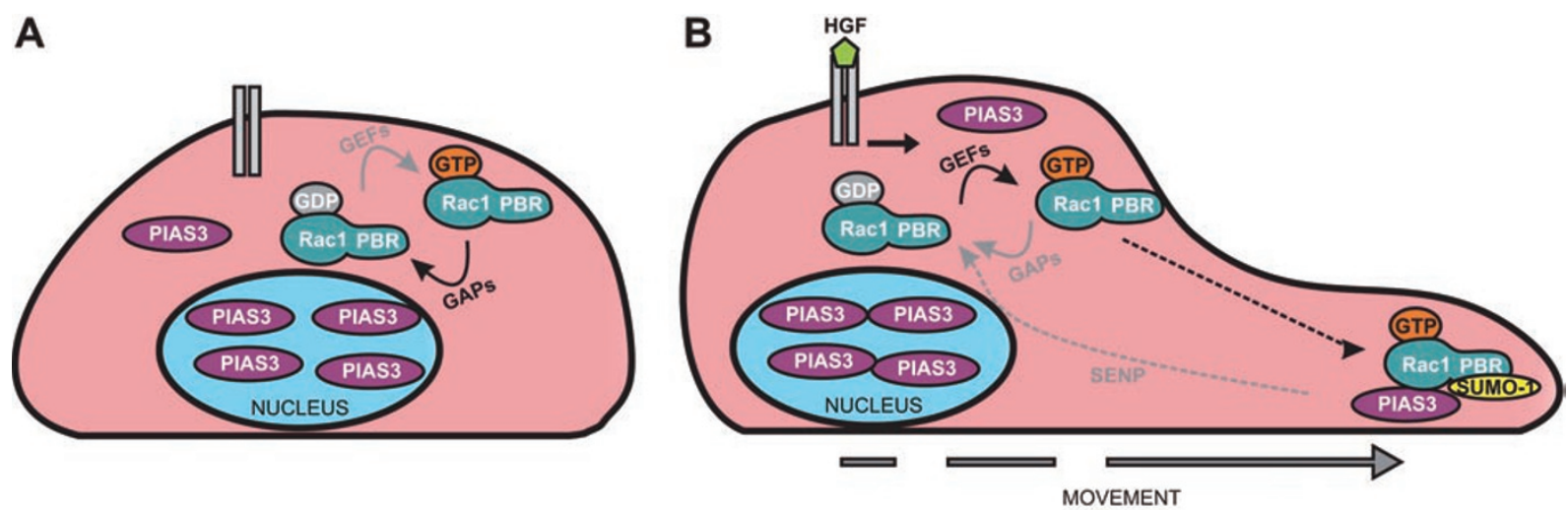

Figure $1 \mathrm{~A}$ summary of the role of PIAS3 and SUMOylation in the regulation of Rac1-dependent cell migration. (A) Situation in a cell in the absence of migration stimulus. (B) HGF signaling through transmembrane receptors induces the GEFs, leading to the activation of Rac1 and PIAS3-enhanced modification of the GTP-Rac1 by SUMO-1, which prolongs the Rac1-GTP activated state, stimulating cell migration.

is the common finding of a very low stoichiometry, i.e. only a small proportion of most of the target proteins are modified at a given time in the cells. In the case of Rac1, interestingly a high proportion of the Rac1-GTP pool was modified $5 \mathrm{~min}$ after the stimulus, but the modification was also highly dynamic; rapidly removed from the protein within 30 min after the stimulus.

As it seems to be the case of an increasing number of SUMO targets, the SUMOylation sites of Rac1 do not conform to the "classic" consensus sequence $\psi$ XKE (hydrophobic amino acid-any amino acid-lysine-glutamic acid). Four lysines (188, 183, 184 and $186)$ in the C-terminal polybasic (PBR) region of Rac1 were identified as potential targets for SUMOylation [6]. Their concomitant mutation abolished the SUMOylation of Rac1 without influencing the ubiquitylation of Rac1. However, the mutation did not affect the interaction with PIAS3 or other Rac1-interacting partners, such as IQGAP, GEFs, $\beta$-Pix or Tiam1. As a consequence of the SUMOylation deficiency, the mutant Rac1 bound less GTP than the wild-type, but the subcellular localization of Rac1 was not altered. The authors performed elegant rescue experiments in Rac1-null mouse embryonic fibroblasts (MEFs) and showed that compared to the wild- type Rac1, the SUMOylation-deficient Rac1 is severely compromised in the lamellipodia-ruffle formation. Moreover, the Rac1 SUMOylation mutant was unable to restore the migration defect or the reduced invasiveness of the Rac1-null MEFs. Importantly, even in the case of the wild-type Rac1, PIAS3 was also required for an efficient rescue of the migration or lamellipodia-ruffle defect. Consequently, the SUMOylation seems to have an important role in the maintenance of the activated Rac1-GTP. How this is achieved remains to be investigated. One possibility is that the interaction of Rac1 with the GEFs and the GAPs is altered due to the SUMO modification of Rac1.

These fascinating data demonstrate for the first time a role for SUMOylation and a PIAS E3 SUMO ligase function in the regulation of Ras superfamily member and cell migration and invasion. The results have ramifications in several diseases, including cancer, and they may in a long run provide translational opportunities for novel therapeutic interventions. As with all first-class research, the current data also provoke several new and important questions. Since the C-terminal PBRs are wellconserved among the Rho/Rac family proteins, it seems likely that a similar SUMOylation- and PIAS-based regula- tion may take place at least with some other Rho/Rac proteins. However, the PIAS protein in charge may be other than the PIAS3, as Castello-Lluva and co-workers did not see any effect in another Rho/Rac family member Cdc42GTP levels upon depletion of PIAS3. Interestingly, in the immediate vicinity of the SUMOylation sites of Rac1 also lies the consensus prenylation site $[10,11]$. The prenylation, covalent addition of geranylgeranyl group to the $\mathrm{C}$-terminus, is known to target the $\mathrm{Rho} / \mathrm{Rac}$ proteins to the cell membrane and promote their activation, perhaps by facilitating their interaction with GEFs. Therefore, an obvious question is whether there is a certain type of crosstalk between the prenylation and the SUMOylation in the regulation of Rac1 function. Even more intriguingly, the PBR of Rac1 has also been reported to harbor a functional nuclear localization signal, which may be regulated through the prenylation $[11,12]$. The PBR may thus function as a switchboard, which coordinates the Rac1 functions, possibly also in the case of other Rho/Rac proteins.

\section{Acknowledgments}

Work in Palvimo laboratory is supported by the Academy of Finland, the Finnish Cancer Organisations and the Sigrid Jusé- 
lius Foundation.

\section{References}

1 Hay RT. SUMO: a history of modification. Mol Cell 2005; 18:1-12.

2 Wilkinson KA, Henley JM. Mechanisms, regulation and consequences of protein SUMOylation. Biochem $J$ 2010; 428:133-145.

3 Rytinki MM, Kaikkonen S, Pehkonen P, Jääskeläinen T, Palvimo JJ. PIAS proteins: pleiotropic interactors associated with SUMO. Cell Mol Life Sci 2009; 66:3029-3041.

4 Johnson ES, Gupta AA. An E3-like factor that promotes SUMO conjugation to the yeast septins. Cell 2001;
106:735-744.

5 Martin S, Nishimune A, Mellor JR, Henley JM. SUMOylation regulates kainate-receptor-mediated synaptic transmission. Nature 2007; 447:321325.

6 Castillo-Lluva S, Tatham MH, Jones $\mathrm{RC}$, et al. SUMOylation of the GTPase Rac1 is required for optimal cell migration. Nat Cell Biol 2010; 12:10781085

7 Heasman SJ, Ridley AJ. Mammalian Rho GTPases: new insights into their functions from in vivo studies. Nat Rev Mol Cell Biol 2008; 9:690-701.

8 Bosco EE, Mulloy JC, Zheng Y. Rac1 GTPase: a "Rac" of all trades. Cell Mol Life Sci 2009; 66:370-374.
9 Parri M, Chiarugi P. Rac and Rho GTPases in cancer cell motility control. Cell Commun Signal 2010; 8:23.

10 Samuel F, Hynds DL. RHO GTPase signaling for axon extension: is prenylation important? Mol Neurobiol 2010; 42:133-142.

11 Williams CL. The polybasic region of Ras and Rho family small GTPases: a regulator of protein interactions and membrane association and a site of nuclear localization signal sequences. Cell Signal 2003; 15:1071-1080.

12 Michaelson D, Abidi W, Guardavaccaro $\mathrm{D}$, et al. Rac1 accumulates in the nucleus during the $\mathrm{G} 2$ phase of the cell cycle and promotes cell division. J Cell Biol 2008; 181:485-496. 